\title{
Opinion
}

\section{Neoantigen: A New Hope for Effective Cancer Immunotherapy}

\author{
Nemat Khansari, PhD* \\ Department of Immunology, School of Medicine, Tehran University of Medical Sciences, Tehran, Iran \\ "Corresponding author \\ Nemat Khansari, PhD \\ Department of Immunology, School of Medicine, Tehran University of Medical Sciences,Tehran, Iran; E-mail: nkhansari928@gmail.com
}

\section{Article Information}

Received: July I2 ${ }^{\text {th }}, 2019 ;$ Accepted: July 22 ${ }^{\text {nd }}, 2019 ;$ Published: July $26^{\text {th }}, 2019$

\section{Cite this article}

Khansari N. Neoantigen:A new hope for effective cancer immunotherapy. Vaccin Res Open J. 2019; 4(I): 19-20. doi: 10.17|40/VROJ-4-II3

$I^{n}$ recent years, immunotherapy with the aim of augmenting the body's immune system to recognize and destroy tumor cells has been widely investigated as a novel cancer therapy modality. Immune responses can be naturally generated against cancer cells. On the other hand, cancer cells can inhibit anticancer immune responses. In the past decades, vaccination with tumor specific/ associated antigens and immunogenic vectors has been successfully used to increase antitumor immune responses. However, the choice of target antigen is imperative in designing a cancer vaccine.

Tumor specific antigens (TSAs) are not expressed in normal cells. TSAs are suitable choices for cancer vaccines. Tumor cells harbor mutations in genes controlling cell growth as well as other genes. Mutations that prevent the repair of deoxyribonucleic acid (DNA) errors that arise as cell divide, this defect called mismatch repair deficiency which has the potential to create neoantigens. These mutations can be contributed to the expression of neoantigens or TSAs. The immune system recognizes TSAs as foreign antigens and immunization with such antigens can induce potent antitumor immune responses. Identification of such TSAs is fundamental for successful cancer immunotherapy. However, identification of TSAs has been difficult, and even impossible, in most cancers due to technical limitations. Recently, technological advances in high-throughput DNA sequencing provided evidences indicating that tumor cells are vastly different from normal cells at the genetic level. ${ }^{1,2}$ These changes lead to expression of unique antigens (neoantigens) in tumor cells. Neoantigens are newly recognized antigens by the immune system. Thus, the immune system can distinguish tumor cells from normal cells and destroy them by recognition of these neoantigens. As neoantigens are only expressed in the tumor cells, mechanisms involved in the induction of immunological tolerance do not hamper antitumor efficacy of neoantigen-based vaccines and such vaccines have lower risk of generating autoimmunity. Development of next-generation DNA sequencing (NGS) technologies facilitates the detection of muta- tions in genes encoding cell surface neoantigens. The number of genes encoding neoantigens in a tumor genome can also be measured by NGS. ${ }^{3}$ In addition, algorithms can be used to predict which of the mutations would create neoantigens. ${ }^{3,4}$ NGS and neoantigen prediction algorithms are beneficial to identify which neoantigens will likely induce an immune response in cancer patients and help to prepare novel vaccines producing $\mathrm{T}$ cell responses to neoantigens.

Recently, identification of neoantigen-reactive tumor infiltrating lymphocytes has been reported from several types of cancers. ${ }^{5}$ It is suggested that neoantigens have been responsible for the clinical efficacy of dendritic cell-based vaccines and adoptive $\mathrm{T}$ cell immunotherapies observed in some cancer patients. Noticeable tumor regressions and the onset of autoimmune melanocyte destruction have been reported in a significant proportion (50-70\%) of patients with metastatic melanoma receiving adoptive transfer of tumor-reactive T cells and high dose IL-2 therapy after a nonmyeloablative lymphodepleting regimen. ${ }^{6,7}$ Tumor exome analysis revealed a neoantigen-specific $\mathrm{T}$ cell reactivity in melanoma. ${ }^{8}$ High-throughput epitope discovery also revealed frequent recognition of neoantigens by $\mathrm{CD}^{+} \mathrm{T}$ cells in melanoma. ${ }^{9}$ Using screening approaches to identify the antigens recognized by clinically effective tumor infiltrating lymphocytes in metastatic cancer patients, it has been found that tumor infiltrating lymphocytes isolated from metastatic cancer patients recognize mutated antigens derived from genes essential for carcinogenesis. ${ }^{10}$

In patients with colorectal, pancreatic, bile duct, and lung cancer, vaccination with a neoantigen peptide, mutated Ras peptide, in combination with IL-2 and/or GM-CSF resulted in induction of immune responses to mutant Ras peptides in 20/37 patients with overall survival of 16.9 -months. ${ }^{11}$ Adoptive transfer of TCR-transduced T cells specific for a mutation-derived neoantigen significantly suppressed the progression of glioma xenografts in mice. ${ }^{12}$ Recently, tumor-infiltrating lymphocytes were expanded 
from human resected ovarian cancer metastases. Autologous somatic mutations were identified with analyses by whole-exome and transcriptome sequencing. All mutated neoepitopes were expressed in antigen presenting cells and then cocultured with TIL fragment cultures. Results showed that mutation-reactive $\mathrm{T}$ cells infiltrated ovarian cancer metastases. ${ }^{13}$ Vaccination of patients with melanoma with a personal neoantigen vaccine targeting 20 predicted personal tumor neoantigens induced polyfunctional $\mathrm{CD} 4^{+}$and $\mathrm{CD} 8^{+} \mathrm{T}$ cells which were able to target $60 \%$ and $16 \%$ of the 97 unique neoantigens used across patients, respectively. Four of six vaccinated patients had no recurrence at 25 -months after vaccination. Two vaccinated patients showed recurrent disease. Subsequent treatment of these two patients with anti-PD-1 therapy resulted in expansion of neoantigen-specific $\mathrm{T}$ cells and complete tumor regression. ${ }^{14}$

Based on my experiences and available data, neoantigen-based vaccines represent a potential new class of cancer immunotherapy. These antigens are usually specific to the tumor of individual patient and provide tumor specific targets for personalized cancer vaccines.

\section{REFERENCES}

1. Schumacher TN, Schreiber RD. Neoantigens in cancer immunotherapy. Science. 2015; 348(6230): 69-74. doi: 10.1126/science. aaa4971

2. Srivastava PK. Neoepitopes of cancers: looking back, looking ahead. Cancer Immunol Res. 2015; 3(9): 969-977. doi: 10.1158/23266066

3. Yuan J, Hegde PS, Clynes R, et al. Novel technologies and emerging biomarkers for personalized cancer immunotherapy. $J$ Immunother Cancer. 2016; 4: 3. doi: 10.1186/s40425-016-0107-3

4. Ma W, Gilligan, BM, Yuan J, Li T. Current status and perspectives in translational biomarker research for PD-1/PD-L1 immune checkpoint blockade therapy. J Hematol Oncol. 2016; 9(1): 47. doi: 10.1186/s13045-016-0277-y

5. Leko V, McDuffie LA, Zheng Z, et al. Identification of neoantigen reactive tumor infiltrating lymphocytes in primarybladder cancer. J Immunol. 2019; 202(12): 3458-3467. doi: 10.4049/jimmunol.1801022
6. Dudley ME, Wunderlich JR, Robbins PF, et al. Cancer regression and autoimmunity in patients after clonal repopulation with antitumor lymphocytes. Science. 2002; 298(5594): 850-854. doi: 10.1126/science.1076514

7. Dudley ME, Yang JC, Sherry R, et al. Adoptive cell therapy for patients with metastatic melanoma: evaluation of intensive myeloablative chemoradiation preparative regimens. J Clin Oncol. 2008; 26(32): 5233-5239. doi: 10.1200/JCO.2008

8. van Roji N, van Burren MM, Philips D, et al. Tumor exome analysis reveals neoantigen specific T-cell reactivity in an ipilimumab-responsive melanoma. J Clin Oncol. 2013; 31(32): e439-e442. doi: 10.1200/JCO.2012.47.7521

9. Linnemann C, van Burren MM, Bies L, et al. High-throughput epitope discovery reveals frequent recognition of neo-antigens by CD4+ T cells in human melanoma. Nat Med. 2015; 21(1): 81-85. doi: $10.1038 / \mathrm{nm} .3773$

10. Lu YC, Yao X, Crystal JS, et al. Efficient identification of mutated cancer antigens recognized by $\mathrm{T}$ cells associated with durable tumor regressions. Clin Cancer Res. 2014; 20(13): 3401-3410. doi: $10.1158 / 1078-0432$

11. Rahma OE, Hamilton JM, Wojtowicz M, et al. The immunological and clinical effects of mutated Ras peptide vaccine in combination with IL-2, GM-CSF, or both in patients with solid tumors. J Transl Med. 2014; 12:55. doi: 10.1186/1479-5876-12-55

12. Chheda ZS, Kohanbash G, Okada K, et al. Novel and shared neoantigen derived from histone 3 variant H3.3K27M mutation for glioma T cell therapy. J Exp Med. 2018; 215(1): 141-157. doi: $10.1084 /$ jem.20171046

13. Deniger DC, Pasetto A, Robbins PF, et al. T-cell responses to TP53 "hotspot" mutations and unique neoantigens expressed by human ovarian cancers. Clin Cancer Res. 2018; 24(22): 5562-5573. doi: 10.1158/1078-0432

14. Ott PA, Hu Z, Keskin DB, et al. An immunogenic personal neoantigen vaccine for patients with melanoma. Nature. 2017; 547(7662): 217-221. doi: 10.1038/nature22991 